\title{
Metastatic Malignant Hemangiopericytoma
}

National Cancer Institute

\section{Source}

National Cancer Institute. Metastatic Malignant Hemangiopericytoma. NCI Thesaurus.

Code C8709.

A malignant hemangiopericytoma which has spread to another anatomical site. 